\title{
BCI Autonomous Assistant System with Seven Tasks for Assisting Disable People
}

\author{
Erdy Sulino Mohd Muslim Tan ${ }^{1}$, Abdul Hamid Adom², Paulraj Murugesa Pandiyan², \\ Sathees Kumar Nataraj ${ }^{2}$, and Marni Azira Markom²
}

\begin{abstract}
Disable person such as a patient who suffer with brain stem stroke and spinal cord injury absolutely need something to live on. Even though they are physically not well or paralyzed, but their brain may work properly. Hence, this paper presents a development of an autonomous system with seven tasks for assisting the patient who is not able to perform their physical but still got their brain to work appropriately. The seven tasks for the system which are concerned to be very important to these people are task for looking for food, drinks and help, make a phone call, assists to toilet, control the television and having rest. Here, the developments of the assistant system with the seven tasks are explained and discussed. In the end, the assistant system is perfectly function and definitely would be able to assist disable community.
\end{abstract}

Keywords - BCI, BMI, Assistant system, EEG.

\section{INTRODUCTION}

$\mathrm{P}$ EOPLE who suffer with intellectual disabilities or physically disable with Motor Neuron Disease (MND), Amyotrophic Lateral Sclerosis (ALS), victims of spinal cord injuries are often paralyzed with voice and mobility impairments. These may cause loss of their voluntary muscle control and they would experience communication difficulties when expressing their needs and thoughts to their caretaker. Today, scientists and engineers are worked together to develop an assistant system that can help these unfortunate people in order to fulfill their necessity. The most popular study in this case is Brain Computer Interface (BCI). Researchers believe that BCI can be applied as an alternative communication system which is it does not rely on muscle function and control. In order to perform this system, electroencephalography (EEG) signals which is used to detect the brain activity when cognitive tasks are performed is employed. Researchers stated that the EEG signal can be measured directly from the cortical surface of the human head to investigate a mental task.

Several successful study and development have been reported such as in [1] by Phillip R. Kennedy. He and his colleagues from Atlanta have pioneered techniques for implanting the neurotrophic electrodes in the cerebral cortex of human patients in 1980s. In 1998, the first patient, who

\footnotetext{
${ }^{1}$ Centre for Diploma Studies, Universiti Malaysia Perlis,Kampus UniCITI Alam,02100 Perlis. MALAYSIA

${ }^{2}$ School of Mechatronics Engineering, Universiti Malaysia Perlis, Pauh Putra, 02700 Perlis, MALAYSIA.
}

was totally paralyzed by a brain stem stroke, had the device implanted and after months of training was able to control a computer cursor by focused thinking [2]. It became the most successful of Kennedy's BCI. Other researcher is Nicolelis and his colleagues [3]. They developed BCIs that decoded brain activity in owl monkeys and used the devices to reproduce monkey movements in systemic arms. Monkeys have advanced reaching and grasping abilities and good hand manipulation skills, making them ideal test subjects for this kind of work.

Based on these success, this paper presents a development of robot assistant for disable person which can perform seven necessary tasks such as looking for food, drinks and help, make a phone call, assist to toilet, control television as well as a relax mode. Also, it is able to arrange pickup and delivery for certain tasks. Hence, it may reduce the burden of the caretakers to a greater extend.

There has two objectives in order to develop this system, i) to develop a BCI system with seven necessary tasks, ii) to develop an assistant system with a mobile robot that able to pick up things and iii) it involves two ways communication, from the human to the robot and from the robot to the human. The system carries a few novelties as state below;

i. Using the designed intelligent communication system (ICS), a neuromuscular disorder person can express his thoughts and communicate with the society very easily.

ii. Simple protocol using only imaginary visual task- easy to remember by users.

iii. The Intelligent communication system (ICS) uses only brain signals to communicate with the society.

iv. Even untrained (naive) users can communicate with the people using the ICS

The developed communication system provides more confident to the ALS subjects and thus it increases the survival period of the subject.

This paper is organized as follow. Section II presents the development of the BCI system with seven tasks. Section III describes the development of BCI assistant system with a mobile robot. Last but not least, Section IV is a conclusion.

\section{DEVELOPMENT OF BCI SySTEM WITH SEVEN TASKS}

Generally, the development of BCI system has a few steps which are included EEG data collection, data pre-processing, feature extraction as well as feature classification. Fig.1 shows the development process of BCI system. 


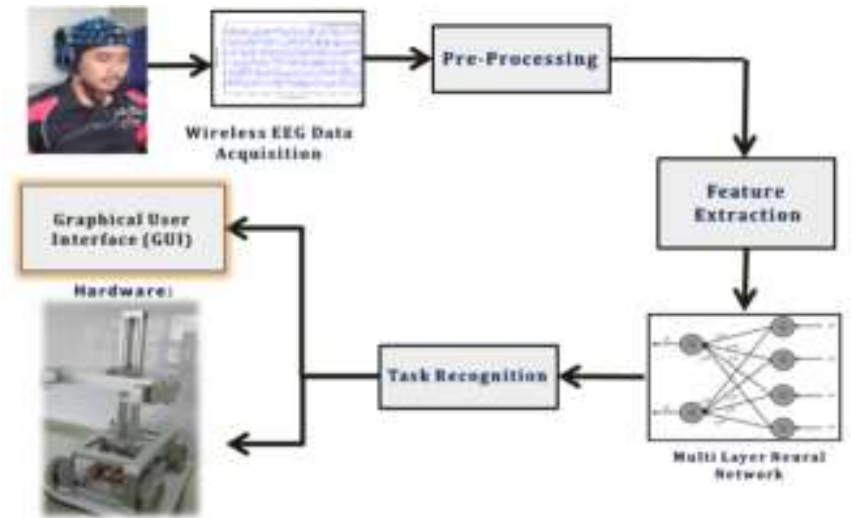

Fig. 1: The BCI system

\section{A. The EEG Signal Amplifier}

The EEG signals are recorded using the EEG signal amplifier which is known as g.MOBIlab. It can record the mental tasks signal into a personal computer [4]. This system is developed by Guger Technologies, Graz, Austria or known as G.tec. Fig. 2 shows the g.MOBIlab amplifier.

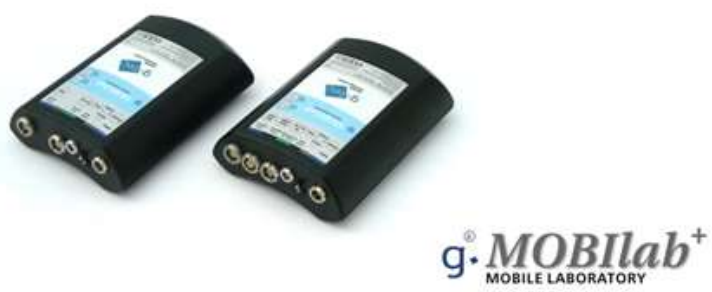

Fig. 2: The g.MOBIlab - biosignal amplifier

According to g.tec, g.MOBIlab+ is the perfect tool for recording multimodal biosignal data on a standard Pocket PC, PC or notebook. This allows investigation of brain, heart and muscle activity, eye movement, respiration, galvanic skin response, pulse and other body signals. The g.MOBIlab+ is available in two versions; i. the 8 channel EEG and, ii. the multi-purpose version. A switch can be connected for external triggering of the data. Also, it is equipped with a low-noise biosignal amplifiers and a 16-bit A/D converter $(256 \mathrm{~Hz})$, which guarantees excellent data quality and a high signal-tonoise ratio. It becomes even more powerful with the software options g.tec provides for Windows and Linux. Programmers can use the C or MATLAB APIs to integrate the device into their own software programs. The collected data can be converted into ASCII-format for other programs like MSExcel or foreign toolboxes.

\section{B. The EEG Electrodes Location}

In this study, the EEG signal is recorded using small gold plated discs electrodes which are placed on the scalp in the motor cortex area in the brain. These positions are identified by the International 10-20 Electrode Placement System as shown in Fig.3 [5]. The electrodes are placed at points that are 10 to 20 percent of these distances. Each electrode is labeled with letter and number. The letter indicated the area of the brain where the electrode is placed and they are shown in TABLE I. The number referred to which side the electrode is placed. If the number is even, hence, the electrode at the right side of the head. Else, it is vice versa. For example, the location of $\mathrm{C} 3$ and $\mathrm{C} 4$, the electrodes are placed at $20 \%$ distance from $\mathrm{Cz}$ location. The $\mathrm{Cz}$ location Is determined by measuring the distance between the nasion and inion for each subject. The earth electrode is located at the F1 location which lies above the right eyebrow.
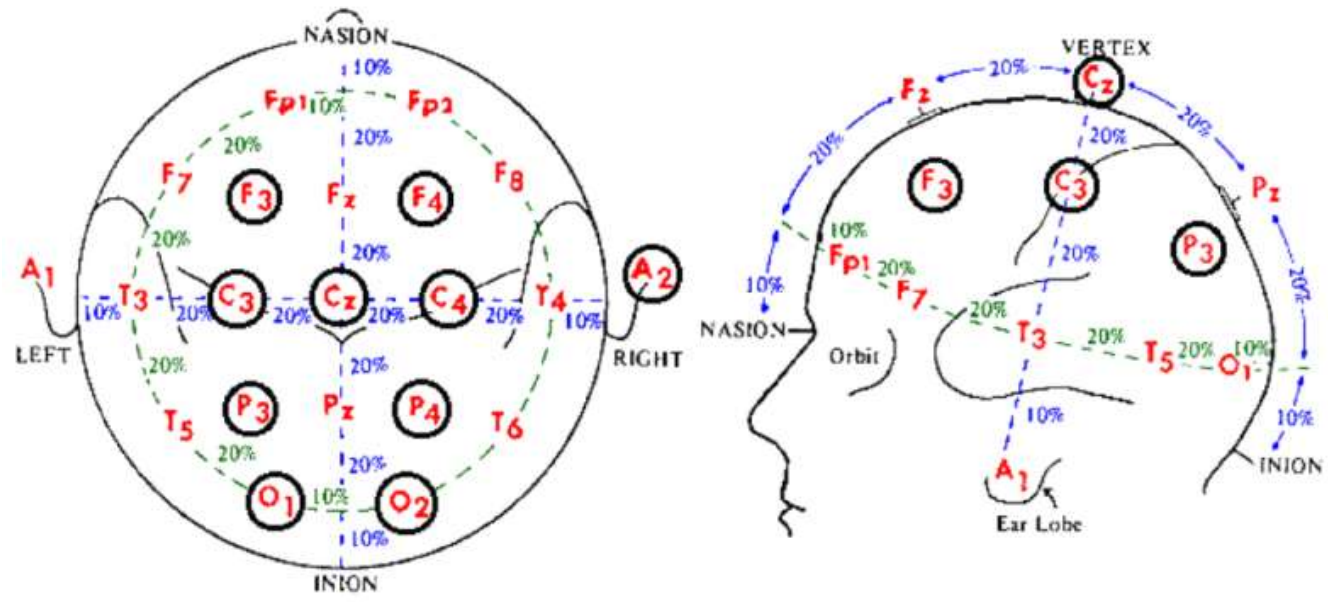

Fig. 3: Electrodes' placement using 10-20 EEG protocols

TABLE I

LETTERS AND THEIR LOCATION IN BRAIN

\begin{tabular}{cc}
\hline \hline Letter & Location in brain \\
\hline $\mathrm{F}$ & Frontal lobe \\
$\mathrm{T}$ & Temporal lobe \\
$\mathrm{C}$ & Central lobe \\
$\mathrm{P}$ & Parietal lobe \\
$\mathrm{O}$ & Occipital lobe \\
\hline \hline
\end{tabular}

\section{The Subjects}

In this study, ten healthy male subjects in the age of 21 to 30 years old are took part who are named as S1, S2, and S3 till S10 are prepared with EEG information, brief description about the purpose and objectives of this research work as well as the outcome of the experiment. The subjects are requested to fill the informed consent form which is included their 
personal details, medical history as well as a little bit of family background. Most of the subjects were students from School of Mechatronic Engineering, Universiti Malaysia Perlis. As advices by medical doctors who are dealing with this disease, they suggested that the subjects must be free from medication and central nervous system abnormalities. Also, the subjects are never been experienced with EEG based communication system [6]. These considerations are taken as requirement because this study is performed for a preliminary work or testing platform before it goes to the real patients. In other words, this study is conducted to show the EEG pattern and profile of the tasks.

\section{D.The Tasks}

For each task, subjects were requested to view the image with a task word which is displayed on the LCD monitor for ten seconds. Then, the LCD monitor is turned on. After that, the subject was requested to imagine the displayed image and pronounce the word mentally. Simultaneously, the EEG signal was recorded for ten seconds during the imagination session. For example, Subject is requested to view an image with 'food'. Then, subject is needed to imagine the image and pronounce the word mentally. Fig. 4 shows the tasks and descriptions.

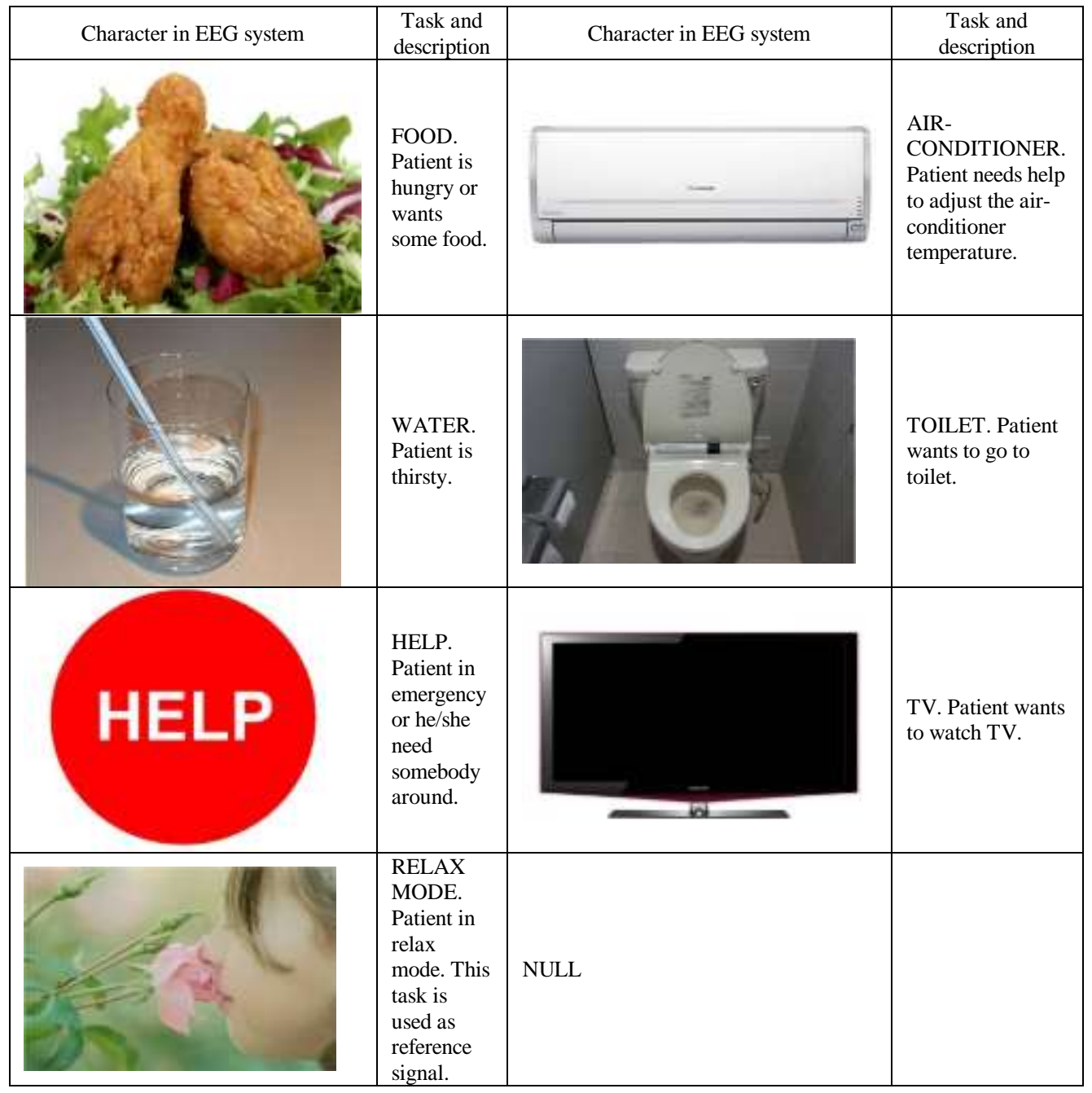

Fig. 4: Tasks for the EEG system and their descriptions

\section{E. The Experiment and Data Collection}

Before starting the work of data collection, the first step that should be considered is the EEG cap. It must be clean by using abrasives electrode gel that give scalp clean from hair gel and dandruff. Conductive paste is used to reduce space and minimize the contact impedance between the electrodes and the head skin. It also serves as a media to ensure lowering of contact impedance at electrode-skin interface. After that, z-check is used to measure the impedance level of each electrode. The impedance values are classified into three levels which are indicated bad, good and best level and they are stated in Table II.

TABLE II

IMPEDANCE LEVEL AND ITS INDICATORS

\begin{tabular}{ccc}
\hline \hline Level & Impedance $(\Omega)$ & Indicator \\
\hline 1 & $1-3$ & Bad \\
2 & $4-6$ & Good \\
3 & $7-10$ & Best \\
\hline \hline
\end{tabular}

The EEG data collection must be performed in quiet and less noise surrounding. This is to provide subjects to be able to focus and concentrate to the tasks given. Any devices that can result electromagnetic field must be shut down or turn off so that its signal does not disturb the EEG data collection performance. This is critical issue because EEG signal may 
always interrupt by any kinds of noise and interferences. Then, subject must wear the EEG cap which is contained the EEG electrodes. He or she must fasten the EEG cap belt at the chest to avoid the EEG cap from moving. The g.MOBIlab and the image monitor are turned on and suppose in standby mode. Basically, a subject took almost a day to complete for all tasks. Subject started with the first task till the seventh task and each task has a rest time about 30 minutes. For each task, signal is recorded for 10 seconds at a sampling frequency of $200 \mathrm{~Hz}$. During the signal recording, initial digital filters are set at $0.1 \mathrm{~Hz}$ to $100 \mathrm{~Hz}$. The collected data were saved in MATLAB file, mat.file, to proceed for preprocessing or data verification process.

\section{F. Data Preprocessing}

In this study, the pre-processing is done by using selection of frequency bands. It has been suggested that frequencies above $40 \mathrm{~Hz}$ convey little information related to mental state [7]. Hence the segmented frame signals are processed using a band pass filter to remove all signals below $0.5 \mathrm{~Hz}$ and above $34 \mathrm{~Hz}$. The segmented brain waves have been categorized into four basic groups; Delta $(0.1-4 \mathrm{~Hz})$, Theta $(4-8 \mathrm{~Hz})$, Alpha $(8-13 \mathrm{~Hz})$ and Beta $(13-30 \mathrm{~Hz})$. Shown in Fig. 6 is data pre-processing result for Task HELP.
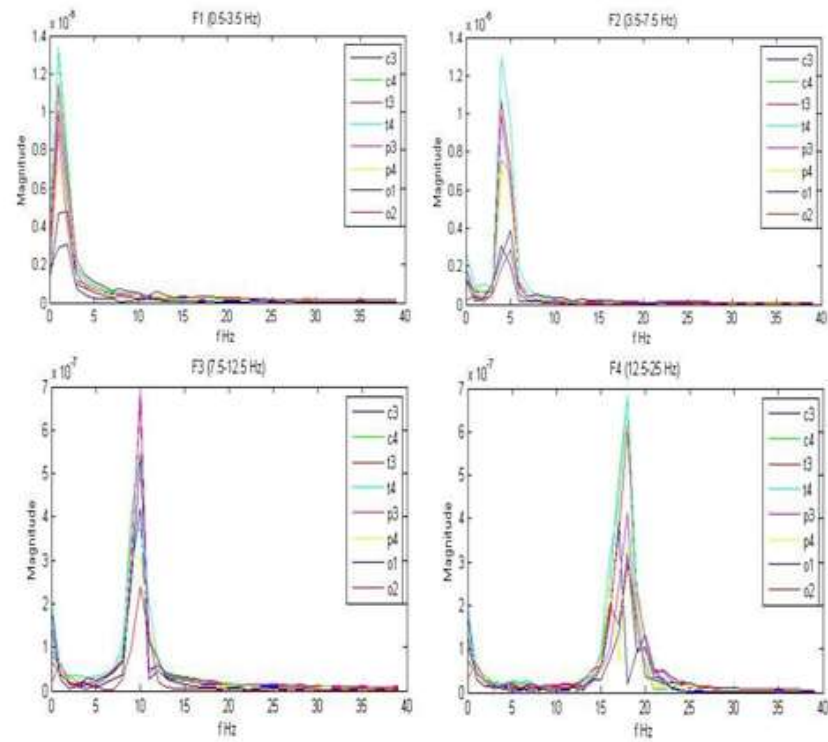

Fig. 6: (a) Spectral band for Delta, (b) Spectral band for Theta, (c) Spectral band for Alpha, and (d) Spectral band for Beta

\section{G. Feature Extraction}

The EEG signal obtained from each channel is divided into frames signals such that each frame has 256 samples. For each frame signal, Bandpass (BP) filters are applied to extract the four frequency band signals. For each band signal, sum of the power values are extracted and a logarithmic transform is performed on the summed power value using Equation (1) and Equation (2) [6]. Therefore for eight channels, this study will have 32 (8 X 4) features per frame.

$$
\text { Band energy } \boldsymbol{B E}=\left[e_{1}, e_{2}, e_{3}, \ldots, e_{i}, \ldots, e_{N}\right]
$$

where, $B \boldsymbol{E}$ is the band energy of the signal and ${ }^{e_{i}}$ is the sum of the squared values of the signals in the $i^{t h}$ frame and it is represented as:

$$
e_{i}=\sum_{j=1}^{256} x_{i j}^{2}
$$

where, frame $(N)$ is 256 and $x_{i j}$ is the instantaneous value of the $j^{\text {th }}$ signal of the $i^{\text {th }}$ frame.

\section{H.Multi Layer Neural Network (MLNN)}

The feature vectors formed is ( $840 \times 32$ feature vectors) system and then associated with the seven tasks profiles vocabulary. Also, the feature vectors are normalized using binary normalization method and partitioned into training set, and testing set. The training set has $(672 \times 32)$ samples and the testing set has the remaining (168 x 32) samples. The MLNN models are activated using logistic sigmoid activation function. The logistic sigmoid function can be scaled to have any range of the values that is appropriate for a given problem. The most common range is from 0.1 to 0.9 . While training the neural network, a Mean Squared Error (MSE) tolerance of 0.1 is used. The learning rate and momentum factor for the models are chosen as 0.1 and 0.8 respectively. The values for learning rate, momentum factor and number of iterations are chosen by experimental observations in order to get better classification accuracy. The predicted task output is compared with the actual imaginary task output and the error is computed. The mean error is then back propagated to the hidden units and the weights are adjusted. This process is repeated until the mean squared error is less than the tolerance value [6]. Thus the output of the network is associated to the corresponding profile tasks, and the task is performed by the assistant system. The MLNN is trained with 25 such trial weights and the number of epoch, network training parameters and the mean classification rate for all the models are shown in Table III.

TABLE III: MLNN MODEL

\begin{tabular}{lc}
\hline \hline Training and testing parameters & Band power feature extraction \\
\hline Training samples & 6720 \\
Number of epochs set & 4000 \\
Number of input neurons & 32 \\
Number of hidden layer & 2 \\
Number of neurons in the 1st layer & 20 \\
Number of neurons in the 2nd layer & 20 \\
Number of output neurons & 3 \\
Training tolerance & 0 \\
\hline \hline
\end{tabular}

\section{MLNN Classification and Confusion Matrix}

Based on TABLE IV, it shows that the MLNN model has a mean epoch of 96 . Then, the network model was trained and obtained a mean training time of 154. The performance of the classification system has a mean accuracy of $95.4 \%$.

A confusion tool is utilised here to visualise the actual and predicted information which is done by classification subsystem. In TABLE V, it shows that the this system can 
obtain 100\% accuracy for the Task 3, Task 6 and Task 7 which contain Task HELP, Task TV and Relax mode respectively. The minimum classification accuracy is $91.67 \%$ which are involve Task Water and Task Air-conditioner.

TABLE IV

MLNN CLASSIFICATION PERFORMANCE

\begin{tabular}{cccccccccc}
\hline \hline \multirow{2}{*}{ Subject Id } & \multicolumn{3}{c}{ Number of Epoches } & \multicolumn{3}{c}{ Training Time (sec) } & \multicolumn{3}{c}{ Classification Rate (\%) } \\
\cline { 2 - 9 } & Min & Mean & Max & Min & Mean & Max & Min & Mean & Max \\
\hline \hline 1 & 36 & 51 & 65 & 65 & 71 & 78 & 94.05 & 95.24 \\
2 & 55 & 99 & 143 & 71 & 185 & 300 & 94.05 & 94.05 & 94.83 \\
3 & 34 & 76 & 121 & 28 & 70 & 87 & 92.86 & 94.64 & 94.64 \\
4 & 45 & 145 & 248 & 61 & 211 & 364 & 92.26 & 93.45 & 94.64 \\
5 & 45 & 93 & 123 & 57 & 148 & 198 & 95.24 & 95.24 & 95.83 \\
6 & 30 & 87 & 143 & 43 & 134 & 225 & 95.83 & 95.83 & 96.43 \\
7 & 124 & 129 & 135 & 135 & 160 & 184 & 96.43 & 97.02 \\
8 & 57 & 102 & 147 & 73 & 191 & 309 & 97.02 & 97.02 & 97.21 \\
9 & 35 & 78 & 125 & 29 & 72 & 90 & 95.83 & 97.62 & 97.62 \\
10 & 46 & 149 & 255 & 63 & 217 & 375 & 95.24 & 96.43 & 97.62 \\
\hline \hline Min & 30 & 51 & 65 & 28 & 70 & 78 & 92.26 & 93.45 & 94.64 \\
\hline Mean & 45 & $\mathbf{9 6}$ & 139 & 62 & $\mathbf{1 5 4}$ & 212 & 95.24 & $\mathbf{9 5 . 5 4}$ & 96.13 \\
\hline Max & 124 & 149 & 255 & 135 & 217 & 375 & 97.02 & 97.62 & 98.21 \\
\hline \hline
\end{tabular}

TABLE V

CONFUSION MATRIX FOR THE BCI ASSISTANT SYSTEM

\begin{tabular}{ccccccccc}
\hline \hline Task & Food & Water & Help & Aircon & Toilet & TV & Relax & $\begin{array}{c}\text { Accuracy } \\
\%\end{array}$ \\
\hline \hline Food & 23 & 0 & 0 & 0 & 0 & 0 & 0 & 95.83 \\
Water & 0 & 22 & 0 & 0 & 0 & 0 & 0 & 91.67 \\
Help & 0 & 0 & 24 & 0 & 0 & 0 & 0 & 100 \\
Aircon & 0 & 0 & 0 & 22 & 0 & 0 & 0 & 91.67 \\
Toilet & 0 & 0 & 0 & 0 & 23 & 0 & 0 & 95.83 \\
TV & 0 & 0 & 0 & 0 & 0 & 24 & 0 & 100 \\
Relax & 0 & 0 & 0 & 0 & 0 & 0 & 24 & 100 \\
\hline \hline
\end{tabular}

\section{Development of BCI Assistant System with Mobile RоBOT}

The development of the assistant system has a few criteria that should be done in order to meet the objectives of the system. The criteria as listed below;

i. It can move autonomously to the specific location that has been program in itself such as to go to a place which has a drink.

ii. It can grip things such as a glass.

iii. It is able to do a task such as get a drink for disable people.

iv. It is able to control a TV remote control and airconditioner.

v. It is able to make a phone call to specific person or caretaker.

vi. It is able to communicate wirelessly with the BCI system which has the results of the classification.

In order to achieve the listed criteria, a mobile robot which is has a grip is developed. The mobile robot is used four motors, DC gear motor, that to enable the robot to move around. A BasicStamp, micro controller is used to program the robot which is containing the instruction to do the listed tasks as above. For the wireless communication or data transmission, a Bluetooth module is used. The mobile robot is shown in Fig. 7.

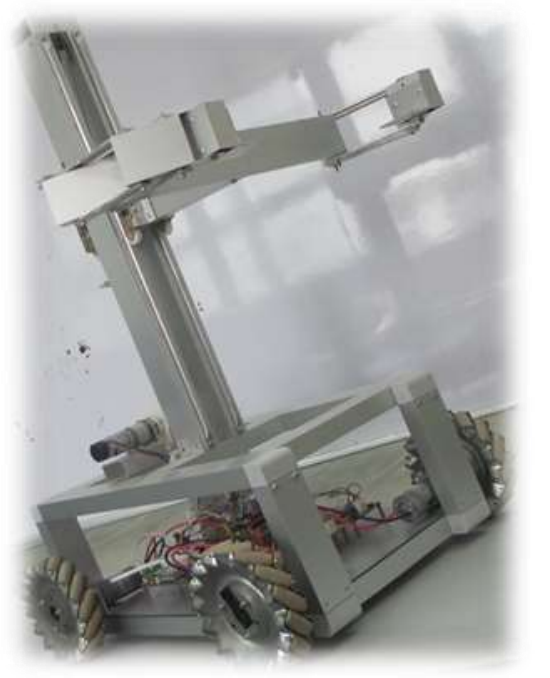

Fig. 7: The mobile robot for the assistant system

\section{CONCLUSION}

Based on the results, the BCI assistant system with mobile system is able to perform with a mean of $96.73 \%$ accuracy. It is a practical percentage value for an EEG system based. Hence, the objectives of this study are completely achieved with utilising of the selection of frequency bands as preprocessing algorithm, band power as feature extraction and MLNN as the training model as well as the classifier. 


\section{ACKNOWLEDGMENT}

Author would like to thank to Prof Dr Hema for her guidance and advices.

\section{REFERENCES}

[1] Kennedy, P.R., Bakay, R.A.E., Moore, M.M., Adams, K. and Goldwaithe, J. (2000) Direct control of a computer from the human central nervous system. IEEE Transactions on Rehabilitation Engineering, Vol 8, Issue 2, pp $198-202$.

http://dx.doi.org/10.1109/86.847815

[2] Kennedy, P.R and Bakay, R.A. (1998) Restoration of neural output from a paralyzed patient by a direct brain connection. Neuro report, 9(8), pp 1707-1711. http://dx.doi.org/10.1097/00001756-199806010-00007

[3] Miguel, A.L. Nicolelis and Chapin, J.K. (2002) Controlling robots with the mind. Scientific American, September 16, 2002.

[4] G.tec medical engineering. (2014) g.MOBIlab+ - g.tec's portable biosignal acquisition and analysis system . Retrieved from http://www.gtec.at/Products/Hardware-and-Accessories/ g.MOBIlabSpecs-Features.

[5] Hema, C.R. (2009) Brain machine interface controlled robot chair. PhD Thesis. Universiti Malaysia Perlis.

[6] Paulraj M P, Abdul Hamid Adom, Sazali Yaacob, Hema C R, Erdy Sulino Mohd Muslim Tan, Sathees Kumar Nataraj (2013) EEG Based Communication System in Generalized \& Customized Modes for Differently Abled Communities. IEESR 2013 Vol 6. Pp 1-13.

[7] McFarland, D.J., Anderson, C.W., Muller, K.R., Schlogl, A. And Krusienski, D.J. (2006) BCI meeting 2005 - Workshop on BCI signal processing: feature extraction and translation. IEEE transaction on neural systems and rehabilitation engineering, 142(2), 135-138. http://dx.doi.org/10.1109/TNSRE.2006.875637 